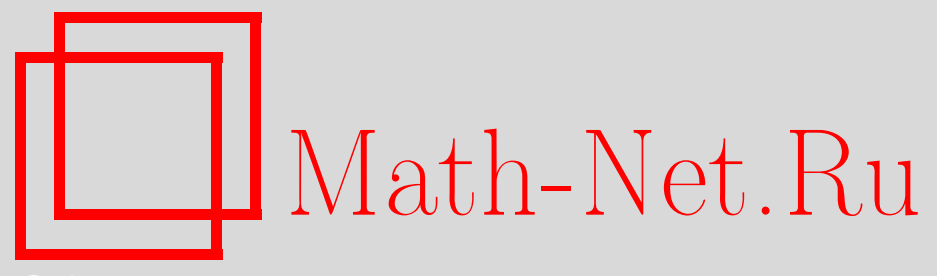

О. А. Логачев, А. А. Сальников, В. В. Ященко, О свойствах сумм Вейля на конечных полях и конечных абелевых группах, Дискрет. матем., 1999, том 11, выпуск 2, 66-85

DOI: https://doi.org/10.4213/dm374

Использование Общероссийского математического портала Math-Net.Ru подразумевает, что вы прочитали и согласны с пользовательским соглашением http://www.mathnet.ru/rus/agreement

Параметры загрузки:

IP: 52.6 .47 .48

26 апреля 2023 г., $15: 26: 38$ 


\title{
О свойствах сумм Вейля на конечных полях и конечных абелевых группах
}

(C) 1999 г. .А. Логачев, А.А. Сальников, В.В. Ященко

\begin{abstract}
Развивается подход, позволяющий привлечь для оценки тригонометрических сумм новые параметры многочленов. Доказывается приведенная оценка Вейля, которая лучше оценки Вейля (в смысле коэффициента при $q^{1 / 2}$ ). Для доказательства вводится новое разбиение всех многочленов на классы эквивалентности такое, что суммы Вейля на каждом классе постоянны. Для произвольной конечной абелевой группы описываются функции, которые являются аналогом многочленов над полем, и для них рассматриваются суммы Вейля.

Работа выполнена при поддержке Российского фонда фундаментальных исследований, проекты 99-01-00929 и 99-01-00941.
\end{abstract}

\section{1. Введение}

Хорошо известно, что ряд, задач теории чисел, теории конечных полей, теории кодирования и криптографии может быть сведен к оценке (как сверху, так и снизу) сумм характеров с полиномиальными аргументами над конечным полем. Нетривиальные верхние оценки тригонометрических сумм над конечными полями были получены в 50-х годах А. Вейлем. Вскоре было показано, что оценка Вейля неулучшаема. При этом имелось в виду, что существует бесконечная последовательность полей и многочленов над ними, для которых оценка Вейля достигается. С более подробными историческими и библиографическими комментариями по проблематике тригонометрических сумм и их приложений можно познакомиться в монографиях $[1,2,3,4]$. В последние годы вопрос достижимости оценки Вейля стал изучаться уже более детально, при разных значениях характеристики поля, степени расширения поля, степеней полиномов (см. $[5,6,7,8])$.

В настоящей работе развивается подход, позволяющий привлечь для оценки тригонометрических сумм новые параметры многочленов. Доказывается приведенная оценка Вейля, которая лучше оценки Вейля (в смысле коэффициента при $q^{1 / 2}$ ). Для доказательства вводится новое разбиение всех многочленов на классы эквивалентности такое, что суммы Вейля на каждом классе постоянны. Для произвольной конечной абелевой группы описываются функции, которые являются аналогом многочленов над полем, и для них рассматриваются суммы Вейля. 


\section{2. Определения; обозначения и результаты}

В обозначениях и исходных результатах мы будем следовать в основном фундаментальной монографии $[2$, т. 1, гл. 5].

Пусть $G$ - конечная абелева группа, $T$ - мультипликативная группа комплексных чисел, по модулю равных 1 , тогда $\widehat{G}$ - группа характеров группы $G$, т.е. группа гомоморфизмов из $G$ в $T$. Если $F_{q}$ - конечное поле характеристики $p$, то для него определены две групповые структуры, аддитивная и мультипликативная, и, соответственно, две группы характеров. Характеры поля $F_{q}$ описываются следующим образом. Пусть $\operatorname{tr}: F_{q} \rightarrow F_{p}-$ функция абсолютного следа из $F_{q}$ в $F_{p}, q=p^{l}, p-$ простое число, определяемая для любого $\alpha \in F_{q}$ равенством

$$
\operatorname{tr}(\alpha)=\alpha+\alpha^{p}+\ldots+\alpha^{p^{l-1}} .
$$

Тогда функция $\chi_{1}$, определяемая для любого $\alpha \in F_{q}$ равенством

$$
\chi_{1}(\alpha)=\exp (2 \pi i \operatorname{tr}(\alpha) / p),
$$

называется каноническим аддитивным характером поля $F_{q}$, здесь $i=\sqrt{-1}$. Все остальные аддитивные характеры поля $F_{q}$ задаются для любых $\alpha, b \in F_{q}$ равенством

$$
\chi_{b}(\alpha)=\chi_{1}(b \alpha) .
$$

Пусть $g-$ некоторый фиксированный примитивный элемент поля $F_{q}$. Тогда все мультипликативные характеры поля $F_{q}$ задаются равенствами

$$
\psi_{j}\left(g^{k}\right)=\exp (2 \pi i j k /(q-1)), \quad k, j=0,1, \ldots, q-2 .
$$

Суммой Вейля на конечном поле $F_{q}$ называется сумма

$$
W_{\chi}(P)=\sum_{x \in F_{q}} \chi(P(x))
$$

где $\chi$ - нетривиальный аддитивный характер поля $F_{q}$, а $P(x) \in F_{q}[x]$ - некоторый многочлен. Кратной суммой Вейля на конечном поле $F_{p}$ будем называть сумму

$$
W_{\chi}(Q)=\sum_{x_{1}, \ldots, x_{l} \in F_{p}} \chi\left(Q\left(x_{1}, \ldots, x_{l}\right)\right)
$$

где $\chi$ - нетривиальный аддитивный характер поля $F_{p}$, а $Q\left(x_{1}, \ldots, x_{l}\right) \in F_{p}\left[x_{1}, \ldots, x_{l}\right]$ - некоторый многочлен.

Аналогично определяется и сумма значений мультипликативного характера (ее тоже можно называть суммой Вейля):

$$
M_{\psi}(P)=\sum_{x \in F_{q}} \psi(P(x))
$$

где $\psi$-расширение мультипликативного характера с множества ненулевых элементов поля на все поле, причем

$$
\psi(0)= \begin{cases}1, & \text { если } \psi \equiv 1, \\ 0 & \text { в противном случае. }\end{cases}
$$


Суммы Вейля имеют многочисленные приложения, но задача точного вычисления этих сумм для многочленов общего положения чрезвычайно сложна. Поэтому обычно ограничиваются оценкой модуля $W_{\chi}(P)$ или вычислением $W_{\chi}(P)$ для некоторых классов многочленов. Тривиальной является оценка

$$
\left|W_{\chi}(P)\right| \leqslant q .
$$

Она достигается на многочленах $P(x)$, для которых $\operatorname{tr}(P(x))$ есть некоторая постоянная. Нетривиальной яв.яется оценка Вейля

$$
\left|W_{\chi}(P)\right| \leqslant(n-1) q^{1 / 2},
$$

доказанная в предположениях, что $P \in F_{q}[x]-$ многочлен степени $n \geqslant 1,(n, q)=1$ и $\chi$ - нетривиальный аддитивный характер. Подчеркнем, что оценка Вейля зависит только от мощности поля и степени многочлена. В известных примерах точного вычисления суммы Вейля используются и другие параметры многочлена, кроме степени. Естественно ожидать, что при уточнении оценки Вейля в нее также будут вводиться новые параметры.

Аналогичная оценка модуля $M_{\psi}(P)$

$$
\left|M_{\psi}(P)\right| \leqslant(m-1) q^{1 / 2}
$$

доказана в предположениях, что $P \in F_{q}[x], P=Q_{1}^{j_{1}} \ldots Q_{k}^{j_{k}}-$ разложение $P$ на неприводимые над $F_{q}$ множители, $m=\operatorname{deg}\left(Q_{1} \ldots Q_{k}\right), \psi$ - нетривиальный мультипликативный характер поля $F_{q}$ показателя $s$ и $\left(s, j_{1}, \ldots, j_{k}\right)=1$.

Свойства сумм Вейля тесно связаны с числом решений уравнения Артина-Шрейepa

$$
y^{q}-y=P(x), \quad P \in F_{q}[x]
$$

и уравнения

$$
\operatorname{tr}_{s}(P(x))=b, \quad P \in F_{q^{s}}[x], \quad b \in F_{q}
$$

где

$$
\operatorname{tr}_{s}(\alpha)=\alpha+\alpha^{q}+\ldots+\alpha^{q^{s-1}}
$$

- относительный след поля $F_{q^{s}}$ над подполем $F_{q}$. Эти уравнения играют особую роль в теории чисел. Приведем один пример такой связи.

Предложение 1. Пусть

$$
\begin{aligned}
W_{s}(P, \chi) & =\sum_{x \in F_{q^{s}}} \chi^{(s)}(P(x)), \\
N_{s}(P, b) & =\left|\left\{x: \operatorname{tr}_{s}(P(x))=b\right\}\right|,
\end{aligned}
$$

где $\chi^{(s)}$ - поднятие характера $\chi$, то есть

$$
\chi^{(s)}(\alpha)=\chi\left(\operatorname{tr}_{s}(\alpha)\right)
$$

Тогда

$$
\begin{aligned}
W_{s}(P, \chi) & =\sum_{b} N_{s}(P, b) \chi(b), \\
N_{s}(P, b) & =\frac{1}{q} \sum_{\chi} W_{s}(P, \chi) \bar{\chi}(b) .
\end{aligned}
$$


Аналогичным образом связаны сумма значений мультипликативного характє и число решений суперэллиптического уравнения

$$
y^{s}=P(x), \quad P \in F_{q}[x]
$$

и уравнения

$$
\operatorname{norm}_{s}(P(x))=b, \quad P \in F_{q^{s}}[x], \quad b \in F_{q},
$$

где

$$
\operatorname{norm}_{s}(\alpha)=\alpha \alpha^{q} \ldots \alpha^{q^{s-1}}
$$

- относительная норма поля $F_{q^{s}}$ над подполем $F_{q}$.

Предложение 2. Пусть

$$
\begin{aligned}
M_{s}(P, \psi) & =\sum_{x \in F_{q^{s}}} \psi^{(s)}(P(x)), \\
K_{s}(P, b) & =\left|\left\{x: \operatorname{norm}_{s}(P(x))=b\right\}\right|,
\end{aligned}
$$

где $\psi^{(s)}$ - поднятие характера $\psi$, то есть

$$
\psi^{(s)}(\alpha)=\psi\left(\operatorname{norm}_{s}(\alpha)\right)
$$

Тогда

$$
\begin{aligned}
M_{s}(P, \psi) & =\sum_{b} K_{s}(P, b) \psi(b), \\
K_{s}(P, b) & =\frac{1}{q} \sum_{\psi} M_{s}(P, \psi) \bar{\psi}(b) .
\end{aligned}
$$

Предложения 1, 2 неявно используются во многих работах, посвященных суммам Вейля (в частности, в [1, стр. 52] и в [2, стр. 386]), поэтому их следует отнести к фольклору. Однако формулировка в явном виде предложений 1,2 позволяет более прозрачно увидеть связь между суммами Вейля и распределением следов значений многочлена.

\section{3. Эквивалентности многочленов, задаваемые тригонометрическими суммами}

Поскольку нас интересуют свойства тригонометрических сумм, можно определить на $F_{q}[x]$ эквивалентности, задаваемые соответствующими суммами, и сократить число многочленов, подлежащих изучению. Вначале сделаем несколько элементарных замечаний.

Во-первых, отметим, что если два многочлена $P_{1}(x), P_{2}(x) \in F_{q}[x]$ задают одно и то же отображение поля $F_{q}$ в себя, то есть $P_{1}(x)=P_{2}(x)$ для любого $x$ из $F_{q}$, то их суммы Вейля также совпадают:

$$
W_{\chi}\left(P_{1}\right)=W_{\chi}\left(P_{2}\right), \quad M_{\psi}\left(P_{1}\right)=M_{\psi}\left(P_{2}\right)
$$


для любых характеров $\chi$ и $\psi$. Это равносильно тому, что суммы Вейля многочленов, сравнимых в кольце $F_{q}[x]$ по идеалу, порождаемому многочленом $x^{q}-x$, совпадают. В частности, степени рассматриваемых многочленов не превышают $q-1$ :

$$
\operatorname{deg} P(x) \leqslant q-1 \text {. }
$$

Во-вторых, при изучении сумм значений аддитивного характера можно ограничиться рассмотрением многочленов степени не выше $q-2$ с нулевым свободным членом. В самом деле, пусть

$$
P(x)=a_{0}+P_{1}(x)+a_{q-1} x^{q-1}, \quad a_{0}, a_{q-1} \in F_{q},
$$

где $\operatorname{deg} P_{1}(x) \leqslant q-2, P_{1}(0)=0$. Ясно, что

$$
\begin{aligned}
W_{\chi}(P) & =\sum_{x \in F_{q}} \chi\left(a_{0}+P_{1}(x)+a_{q-1} x^{q-1}\right) \\
& =\chi\left(a_{0}\right)\left(\chi(0)+\sum_{x \in F_{q}^{*}} \chi\left(P_{1}(x)+a_{q-1} x^{q-1}\right)\right) \\
& =\chi\left(a_{0}\right)+\chi\left(a_{0}+a_{q-1}\right) \sum_{x \in F_{q}^{*}} \chi\left(P_{1}(x)\right) \\
& =\chi\left(a_{0}\right)-\chi\left(a_{0}+a_{q-1}\right)+\chi\left(a_{0}+a_{q-1}\right) \sum_{x \in F_{q}} \chi\left(P_{1}(x)\right) .
\end{aligned}
$$

Таким образом, показано, что

$$
W_{\chi}(P)=c+d W_{\chi}\left(P_{1}\right)
$$

где $c=\chi\left(a_{0}\right)-\chi\left(a_{0}+a_{q-1}\right)$ и $d=\chi\left(a_{0}+a_{q-1}\right)$.

Обозначим через $\mathscr{K}^{\prime} \subset F_{q}[x]$ множество многочленов степени не выше $q-2$ с нулевым свободным членом.

Теперь рассмотрим менее тривиальную эквивалентность: будем называть многочлены $P_{1}(x)$ и $P_{2}(x)$ эквивалентными по следу, если для всех $x \in F_{q}$

$$
\operatorname{tr}\left(P_{1}(x)\right)=\operatorname{tr}\left(P_{2}(x)\right) .
$$

Поскольку $\operatorname{tr}$ - линейное отображение, т. е. гомоморфизм аддитивной группы кольца $F_{q}[x]$, то многочлены $P_{1}(x)$ и $P_{2}(x)$ эквивалентны по следу тогда и только тогда, когда $P_{1}(x)-P_{2}(x)$ принадлежит ядру указанного гомоморфизма. Как известно (см., например, $[1$, стр. 32$]), P(x)$ принадлежит этому ядру тогда и только тогда, когда для каждого $x \in F_{q}$ он может быть представлен в виде $P(x)=\Phi(x)-\Phi^{p}(x)$ с некоторым $\Phi(x) \in F_{q}[x]$. Теперь опишем образ гомоморфизма tr. Зафиксируем некоторый базис поля $F_{q}$ и будем рассматривать $F_{q}$ как векторное пространство над $F_{p}$. Поскольку значения $\operatorname{tr} P(x)$ принадлежат простому полю $F_{p}$, очевидно, что

$$
\operatorname{tr} P(x) \in F_{p}\left[x_{1}, \ldots, x_{l}\right] .
$$

С другой стороны, для каждого многочлена $Q \in F_{p}\left[x_{1}, \ldots, x_{l}\right]$ можно построить такой многочлен $P \in F_{q}^{\prime}[x]$, что

$$
\operatorname{tr} P(x)=Q\left(x_{1}, \ldots, x_{l}\right) .
$$

Теперь воспользуемся теоремой о гомоморфизмах и получим следующее утверждение. 
Теорема 1. Факторгруппа аддитивной группъ колъца $F_{q}[x]$ по ядру гомоморфизма $\operatorname{tr}: F_{q}[x] \rightarrow F_{p}\left[x_{1}, \ldots, x_{l}\right]$ изоморфна аддитивной группе колъца $F_{p}\left[x_{1}, \ldots, x_{l}\right]$.

Следствие 1. Множество значений сумм Вейля $W_{\chi}(P)$, когда многочлен $P$ пробегает кольцо $F_{q}[x]$, совпадает с множеством значений кратных сумм Вейля $W_{\chi}(Q)$, когда многочлен $Q$ пробегает кольцо $F_{p}\left[x_{1}, \ldots, x_{l}\right]$.

Классы эквивалентности многочленов по следу - это в точности смежные классы аддитивной группы $F_{q}[x]$ по ядру гомоморфизма $\operatorname{tr}$ и они находятся во взаимно однозначном соответствии с многочленами из $F_{p}\left[x_{1}, \ldots, x_{l}\right]$. Опишем один способ выбора в каждом смежном классе по одному представителю специального вида.

Напомним предварительно понятие циклотомического класса [9, стр. 108]. Множество вычетов по модулю $p^{l}-1$ относительно операции умножения на $p$ распадается на подмножества, называемые циклотомическими классами по модулю $p^{l}-1$. Циклотомический класс $C_{s}$, в котором $s$ - наименьшее число, состоит из чисел

$$
\left\{s, s p, s p^{2}, \ldots, s p^{l_{s}-1}\right\},
$$

где $l_{s}-$ наименьшее целое положительное число такое, что $s p^{l_{s}} \equiv s\left(\bmod p^{l}-1\right)$. Число $s$ будем называть представителем циклотомического класса $C_{s}$. Множество всех ненулевых представителей обозначим $\Pi_{l}$.

Мощность $l_{s}$ циклотомического класса $C_{s}$ делит число $l$, поэтому в поле $F_{q}$ существует (единственное) подполе $F_{p^{l}}$ порядка $p^{l_{s}}$. Относительный след поля $F_{q}$ над полем $F_{p^{l_{s}}}$ - это линейное отображение, ядро которого состоит из элементов вида $b-b^{p^{l s}}, b \in F_{q}$. В каждом смежном классе аддитивной группы поля $F_{q}$ по этому ядру имеется единственный элемент вида $c_{s} \zeta_{s}, c_{s} \in F_{p^{l}}, \zeta_{s}-$ некоторый фиксированный элемент из $F_{q}$, относительный след которого отличен от нуля. Если $l / l_{s}$ не делится на $p$, то в качестве $\zeta_{s}$ можно взять единицу.

Многочлен $\Phi(x) \in \mathscr{K}$ будем называть циклотомически приведенным, или $c r-$ многочленом, если он имеет вид

$$
\Phi(x)=\sum_{s \in \Pi_{l}} c_{s} \zeta_{s} x^{s}, \quad c_{s} \in F_{p^{l s}}
$$

Теорема 2. Каждый многочлен из $\mathscr{K}$ эквивалентен по следу некоторому чиклотомически приведенному многочлену.

Доказательство. Пусть

$$
P(x)=\sum_{i=1}^{p^{l}-2} a_{i} x^{i} \in \mathscr{K}
$$

Представим $P(x)$ в виде

$$
P(x)=\sum_{s \in \Pi_{l}} \sum_{j=0}^{l_{s}-1} a_{s p^{j}} x^{s p^{j}}=\sum_{s \in \Pi_{l}} P_{s}(x) .
$$

Зафиксируем $s \in \Pi_{l}$ и рассмотрим многочлен $P_{s}(x)$, который естественно назвать циклотомически однородным:

$$
P_{s}(x)=\sum_{j=0}^{l_{s}-1} a_{s p^{j}} x^{s p^{j}}
$$


Подберем такой многочлен

$$
\Phi_{s}(x)=\sum_{j=0}^{l_{s}-1} b_{s p^{j}} x^{s p^{j}}
$$

и такой элемент $c_{s} \in F_{p^{!} s}$, что

$$
P_{s}(x)+\Phi_{s}(x)-\Phi_{s}^{p}(x)=c_{s} \zeta_{s} x^{s}
$$

Это будет означать, что следы многочленов $P_{s}(x)$ и $c_{s} \zeta_{s} x^{s}$ при любом $x$ из $F_{q}$ совпадают, или, что многочлены $P_{s}(x)$ и $c_{s} \zeta_{s} x^{s}$ эквивалентны. Уравнение (1) равносильно системе

$$
\begin{aligned}
a_{s}+b_{s}-b_{s p^{l_{s}-1}}^{p} & =c_{s} \zeta_{s}, \\
a_{s p}+b_{s p}-b_{s}^{p} & =0, \\
\cdots & \\
a_{s p^{l_{s}-1}}+b_{s p^{l_{s}-1}}-b_{s p^{l_{s}-2}}^{p} & =0 .
\end{aligned}
$$

Выберем элемент $b_{s}=b \in F_{q}$ произвольным образом. Тогда остальные коэффициенты $b_{s p^{j}}$ и элемент $c_{s}$ определяются из системы (2) однозначно:

$$
\begin{aligned}
b_{s p} & =-a_{s p}+b^{p} \\
b_{s p^{2}} & =-a_{s p^{2}}+b_{s p}^{p}=-a_{s p^{2}}-a_{s p}^{p}+b^{p^{2}} \\
& \ldots \\
b_{s p^{l_{s}-1}} & =-a_{s p^{l_{s}-1}}+b_{s p^{l_{s}-2}}^{p}-a_{s p^{l_{s}-1}}-\ldots-a_{s p}^{p^{l_{s}-2}}+b^{p^{l_{s}-1}} \\
c_{s} \zeta_{s} & =a_{s}+b-b_{s p^{l_{s-1}}}^{p}=a_{s}+\ldots+a_{s p}^{p^{l_{s}-1}}+b-b^{p^{l_{s}}} .
\end{aligned}
$$

Отсюда видно, что когда элемент $b$ пробегает поле $F_{q}$, элемент $c_{s} \zeta_{s}$ пробегает фиксированный, в зависимости от $a_{s}+a_{s p^{l_{s-1}}}^{p}+\ldots+a_{s p}^{p^{l_{s-1}}}$, смежный класс аддитивной группы поля $F_{q}$ по ядру относительного следа по подполю $F_{p^{l_{s}}}$. Поэтому можно выбрать $b \in F_{q}$ так, что $c_{s} \in F_{p^{l_{s}}}$.

Проделав эти вычисления для каждого $s \in \Pi_{l}$, получим искомое утверждение.

То, что при изучении тригонометрических сумм достаточно рассматривать многочлены, у которых от нуля отличны лишь коэффициенты при степенях, являющихся минимальными представителями циклотомических классов, специалисты понимали и ранее (см., например, [10], где такие многочлены называются каноническими). Теорема 2 показывает, что в ряде случаев можно дополнительно сократить множество рассматриваемых многочленов за счет специального выбора коэффициентов при таких степенях. В частности, два различных канонических многочлена могут оказаться эквивалентными по следу. Непонимание этого приводит к ошибкам (см. [10, лемма 1]).

Пример 1. Над конечным полем $F_{q}$, где $q=p^{l}, p$ - простое число и $l=l_{1} l_{2}$, причем $l_{1}, l_{2} \geqslant 2$, рассмотрим одночлен $P(x)=a x^{s}$. При этом

$$
s=\frac{p^{l_{1} l_{2}}-1}{p^{l_{2}}-1}, \quad a=\xi^{p^{l_{2}}}-\xi,
$$


где $\xi$ - примитивный элемент поля $F_{q}$. Легко проверить, что $s$ является минимальным представителем своего циклотомического класса и

$$
s p^{l_{2}} \equiv s \quad(\bmod q-1) .
$$

Очевидно, что $a \neq 0$. Однако, поскольку $\operatorname{tr} P(x)=\operatorname{tr} P^{p}(x)$, одночлен $P(x)$ эквивалентен по следу нулю:

$$
\operatorname{tr} P(x)=\operatorname{tr}\left(\xi^{l_{2}} x^{s}\right)-\operatorname{tr}\left(\xi x^{s}\right)=\operatorname{tr}\left(\left(\xi x^{s}\right)^{p^{l_{2}}}\right)-\operatorname{tr}\left(\xi x^{s}\right)=0 .
$$

Аналогичным образом исследуем эквивалентность многочленов, задаваемую функцией норма: будем называть многочлены $P_{1}(x)$ и $P_{2}(x)$ эквивалентными по норме, если для всех $x \in F_{q}$

$$
\operatorname{norm}\left(P_{1}(x)\right)=\operatorname{norm}\left(P_{2}(x)\right) .
$$

Как известно (см., например, $\left[1\right.$, стр. 32]), $\operatorname{norm}(P(x))=1$ для всех $x \in F_{q}$ тогда и только тогда, когда многочлен $P(x)$ может быть представлен для каждого $x \in F_{q}$ в виде $P(x)=\Phi^{1-p}(x)$ с некоторым многочленом $\Phi(x) \in F_{q}[x]$, не имеющим корней в поле $F_{q}$. Поэтому

$$
\operatorname{norm}\left(P_{1}(x)\right)=\operatorname{norm}\left(P_{2}(x)\right)
$$

для всех $x \in F_{q}$ тогда и только тогда, когда для всех $x \in F_{q}$

$$
P_{2}(x)=\Phi^{1-p}(x) P_{1}(x),
$$

где многочлен $\Phi(x) \in F_{q}[x]$ не имеет корней в поле $F_{q}$. В частности, различные неприводимые над $F_{q}$ многочлены степени не выше $q-2$ не эквивалентны по норме. Отсюда, пользуясь мультипликативностью нормы и однозначностью разложения любого многочлена в произведение неприводимых, легко получить следующую теорему.

Теорема 3. Пусть $P \in F_{q}[x], P=Q_{1}^{j_{1}} \ldots Q_{k}^{j_{k}}-$ разложение $P$ на неприводимъе над $F_{q}$ множители и

$$
j_{r}^{\prime}= \begin{cases}j_{r}(\bmod p-1), & \text { если } j_{r} \quad(\bmod p-1) \leqslant(p-1) / 2, \\ p-1-j_{r}(\bmod p-1) & \text { в противном случае }\end{cases}
$$

причем $j_{r}^{\prime} \neq 0$ при $r=1, \ldots, t u j_{r}^{\prime}=0$ при $r=t+1, \ldots, k$. Многочлен $P(x)$ и все эквивалентные ему по норме многочлены эквивалентны многочлену $P^{\prime}=$ $Q_{1}^{j_{1}^{\prime}} \ldots Q_{t}^{j_{t}^{\prime}}$

В соответствии с теоремой 2 корректным является следующее обозначение: для любого многочлена $P(x) \in \mathscr{K}$ через $\operatorname{Deg} P$ обозначим степень нелинейности $c r-$ многочлена, эквивалентного по следу данному многочлену $P(x)$. Очевидно, что для любого $P(x) \in \mathscr{K}$

\section{$\operatorname{Deg} P \leqslant \operatorname{deg} P$}

и число $\operatorname{Deg} P$ взаимно просто с $p$. Отметим, что спектр значений $\operatorname{Deg} P$ для многочленов $P(x) \in \mathscr{K}$ меньше, чем спектр значений $\operatorname{deg} P$. В частности, число различных значений, которые может принимать $\operatorname{Deg} P$, совпадает с числом циклотомических классов. Последовательное применение теоремы 2 и оценки Вейля позволяет улучшить оценку Вейля. 
Теорема 4 (приведенная оценка Вейля). Пустъ $P(x)$ - многочлен из колъца $F_{q}[x]$ степени не выие $q-2$ с нулевъм свободным иленом $и \chi-$ нетривиалъный аддитивный характер поля $F_{q}$. Тогда

$$
\left|\sum_{x \in F_{q}} \chi(P(x))\right| \leqslant(\operatorname{Deg} P-1) q^{1 / 2},
$$

ecлu $\operatorname{Deg} P \neq 0 u$

$$
\left|\sum_{x \in F_{q}} \chi(P(x))\right|=q
$$

если $\operatorname{Deg} P=0$.

Доказательство. Если $\operatorname{Deg} P=0$, то утверждение теоремы очевидно. Предположим, что $\operatorname{Deg} P \neq 0$. Применим к многочлену $P(x)$ теорему 2 и построим $c r$-многочлен $\widetilde{P}(x)$ степени нелинейности $\operatorname{Deg} P$ такой, что

$$
\operatorname{tr} P(x)=\operatorname{tr} \widetilde{P}(x)
$$

для любого $x \in F_{q}$. Тогда, для любого характера $\chi$

$$
W_{\chi}(P)=W_{\chi}(\widetilde{P})
$$

Поскольку $\operatorname{Deg} P$ взаимно просто с $p$, к многочлену $\widetilde{P}(x)$ можно применить оценку Вейля и получить требуемое неравенство

$$
\left|W_{\chi}(P)\right|=\left|W_{\chi}(\widetilde{P})\right| \leqslant(\operatorname{Deg} P-1) q^{1 / 2}
$$

В приведенной оценке Вейля используется новый параметр многочлена, Deg $P$, вычисление которого для конкретного многочлена $P$, очевидно, не представляет особого труда. При этом оценка Вейля улучшается в трех направлениях:

(1) расширяется область применимости оценки, так как нет требования взаимной простоты $\operatorname{deg} P$ и $p$;

(2) расширяется область, в которой оценка эффективнее тривиальной, так как очевидно, что в ряде случаев одновременно выполняются оба неравенства, $\operatorname{deg} P \geqslant q^{1 / 2}$ и $\operatorname{Deg} P \leqslant q^{1 / 2}$

(3) как правило, $\operatorname{Deg} P<\operatorname{deg} P$, поэтому приведенная оценка точнее; более того, как показывают примеры, отношение $\operatorname{deg} P / \operatorname{Deg} P$ может иметь порядок даже $q^{\varepsilon}$

Наиболее наглядно этот эффект виден на примере циклотомически однородного многочлена, который в соответствии с теоремой 2 эквивалентен одночлену. Пусть для некоторого $s \in \Pi_{l}$

$$
P_{s}(x)=\sum_{j=0}^{l_{s}-1} a_{s p^{j}} x^{s p^{j}}
$$


тогда многочлен $P_{s}(x)$ эквивалентен $c_{s} \zeta_{s} x^{s}$ для некоторого $c_{s} \in F_{p^{l}}$, зависящего от $a_{s p^{j}}, j=0, \ldots, l_{s}-1$. Если $a_{s p^{l-1}}=\ldots=a_{s p^{k+1}}=0, \quad a_{s p^{k}} \neq 0$, то $\operatorname{Deg} P=s p^{k}$, и если $c_{s} \neq 0$, то $\operatorname{Deg} P=s$. В этом случае

$$
\frac{\operatorname{deg} P}{\operatorname{Deg} P}=p^{k}, \quad 0 \leqslant k \leqslant l_{s}-1
$$

В качестве следствия теоремы 4 можно сформулировать такое утверждение: необходимым условием достижимости обычной оценки Вейля для многочлена $P(x)$ является равенство $\operatorname{deg} P=\operatorname{Deg} P$.

Аналогичными рассуждениями, путем последовательного применения теоремы 3 и оценки Вейля для сумм значений мультипликативного характера можно улучшить эту оценку.

Теорема 5. Пусть $P \in F_{q}[x], P=Q_{1}^{j_{1}} \ldots Q_{k}^{j_{k}}-$ разложение $P$ на неприводимъе над $F_{q}$ множители и

$$
j_{r}^{\prime}= \begin{cases}j_{r}(\bmod p-1), & \text { если } j_{r} \quad(\bmod p-1) \leqslant(p-1) / 2 \\ p-1-j_{r}(\bmod p-1) & \text { в противном случае }\end{cases}
$$

причем $j_{r}^{\prime} \neq 0$ nрu $r=1, \ldots, t u j_{r}^{\prime}=0$ nрu $r=t+1, \ldots, k$. Положим $m^{\prime}=$ $\operatorname{deg}\left(Q_{1} \ldots Q_{t}\right)$. Пусть $\psi$ - нетривиалъный мулътипликативный характер поля $F_{q}$ показателя $s$ u $\left(s, j_{1}^{\prime}, \ldots, j_{t}^{\prime}\right)=1$. Тогда

$$
\left|\sum_{x \in F_{q}} \psi(P(x))\right| \leqslant\left(m^{\prime}-1\right) q^{1 / 2}
$$

Развитие подхода, предложенного в [5], с помощью следствия 1 и теоремы 2 позволяет получить новые оценки для сумм Вейля. Для этого введем, следуя [5], некоторые обозначения. Положим

$$
S(n, q)=\max \left|W_{\chi}(P)\right|, \quad n \leqslant q-2,
$$

где максимум берется по всем многочленам $P(x) \in F_{q}[x]$ без свободного члена степени не выше $n$ таким, что $\operatorname{tr}(P(x)) \neq c$, где $c-$ постоянная. Аналогично, положим

$$
S_{l}(t, p)=\max \left|W_{\chi}(Q)\right|,
$$

где максимум берется по всем многочленам $Q\left(x_{1}, \ldots, x_{l}\right) \in F_{p}\left[x_{1}, \ldots, x_{l}\right]$ без свободного члена степени не выше $t$. Назовем $p$-ичным весом числа $n \in\left\{0,1, \ldots, p^{l}-2\right\}$ сумму цифр $p$-ичного представления числа $n$. Если обозначить $p$-ичный вес через $\|n\|_{p}$, то

$$
\|n\|_{p}=\sum_{i=0}^{l-1} n_{i}
$$

при

$$
n=\sum_{i=0}^{l-1} n_{i} p^{i}, \quad 0 \leqslant n_{i} \leqslant p-1
$$


Известно, что $p$-ичный вес постоянен на циклотомических классах по модулю $p^{l}-1$. Ясно, что

$$
\|n\|_{p} \leqslant(p-1)\left\lceil\log _{p} n\right\rceil-1 \leqslant l(p-1)-1,
$$

где $\lceil a\rceil$ - наименьшее целое число, большее либо равное $a$.

Рассмотрим все циклотомические классы, $p$-ичный вес на которых не превосходит $t$, и обозначим через $n_{p}(t, l)$ максимального из представителей этих циклотомических классов. Рассмотрим минимальный набор циклотомических классов, который содержит все числа от 1 до $n$, и обозначим через $\pi_{p}(n, l)$ максимального из представителей этих циклотомических классов. Ясно, что $1 \leqslant \pi_{p}(n, l) \leqslant n$.

Качественно введенные обозначения выражают следующее: $\pi_{p}(n, l)$ - это максимальная степень $c r$-многочленов, эквивалентных всем многочленам степени не выше $n$. Циклотомические классы и $p$-ичный вес появляются при переходе в соответствии с теоремами 1 и 2 от многочленов из $F_{q}[x]$ к многочленам из $F_{p}\left[x_{1}, \ldots, x_{l}\right]$ и к $c r$-многочленам. В частности, максимальная степень многочленов из $F_{p}\left[x_{1}, \ldots, x_{l}\right]$, которые могут быть при этом получены из многочленов степени не выше $n$ в $F_{q}[x]$, не превосходит $(p-1)\left\lceil\log _{p} n\right\rceil$. При обратном переходе к $c r$-многочленам от одной переменной аналогичный смысл имеет $n_{p}(t, l)$.

Лемма 1. Справедливо равенство

$$
S\left(n, p^{l}\right)=S\left(\pi_{p}(n, l), p^{l}\right) .
$$

Доказательство. Из теоремы 2 вытекает, что множество значений сумм Вейля $W_{\chi}(P)$, когда многочлен $P$ пробегает некоторое множество $\mathscr{M} \subset F_{q}[x]$, совпадает с множеством значений сумм Вейля $W_{\chi}\left(P_{1}\right)$, когда $P_{1}$ пробегает множество $c r-$ многочленов, эквивалентных многочленам из $\mathscr{M}$. Из этого, в частности, и вытекает лемма 1.

При $n>p^{l / 2}$ с помощью леммы 1 можно построить даже серию равенств вида

$$
S\left(n_{0}, p^{l}\right)=S\left(n_{0}-1, p^{l}\right)=\ldots=S\left(n_{0}-k_{0}, p^{l}\right)
$$

при некоторых $n_{0}, k_{0}$.

Теорема 6. Величины $S\left(n, p^{l}\right)$ u $S_{l}(t, p)$ связаны соотношениями

$$
\begin{aligned}
S\left(n_{p}(t, l), p^{l}\right) & \geqslant S_{l}(t, p), \\
S\left(n, p^{l}\right) & \leqslant S_{l}\left((p-1)\left\lceil\log _{p} n\right\rceil-1, p\right) .
\end{aligned}
$$

Доказательство. Неравенство (3) доказано в [5]. Из теорем 1, 2 и следствия 1 оно получается с помощью следующего рассуждения. Выберем некоторый многочлен $Q\left(x_{1}, \ldots, x_{l}\right) \in F_{p}\left[x_{1}, \ldots, x_{l}\right]$ степени не выше $t$, для которого

$$
\left|W_{\chi}(Q)\right|=S_{l}(t, p)
$$

Из приведенных выше результатов вытекает, что найдется такой многочлен $P(x)$ из $F_{q}[x]$ степени не выше $n_{p}(t, l)$, для которого

$$
W_{\chi}(P)=W_{\chi}(Q) .
$$


Но по определению $S\left(n_{p}(t, l), p^{l}\right)$

$$
\left|W_{\chi}(P)\right| \leqslant S\left(n_{p}(t, l), p^{l}\right)
$$

поэтому неравенство (3) вытекает из (5), (6), (7).

Аналогичными рассуждениями получается неравенство (4). Выберем некоторый многочлен $P(x) \in F_{q}[x]$ степени не выше $n$, для которого

$$
\left|W_{\chi}(P)\right|=S\left(n, p^{l}\right)
$$

Найдется многочлен $Q\left(x_{1}, \ldots, x_{l}\right) \in F_{p}\left[x_{1}, \ldots, x_{l}\right]$ степени не выше $(p-1)\left\lceil\log _{p} n\right\rceil$, для которого

$$
W_{\chi}(Q)=W_{\chi}(P)
$$

Но по определению $S_{l}\left((p-1)\left\lceil\log _{p} n\right\rceil-1, p\right)$

$$
\left|W_{\chi}(Q)\right| \leqslant S_{l}\left((p-1)\left\lceil\log _{p} n\right\rceil-1, p\right),
$$

поэтому неравенство (4) вытекает из (8), (9), (10).

Переход от величин $S\left(n, p^{l}\right)$ к величинам $S_{l}(t, p)$ удобен по нескольким причинам. В частности, для некоторых $p$ величины $S_{l}(t, p)$ известны из других соображений. Кроме того, для величин $S_{l}(t, p)$ можно установить рекуррентные по $l$ неравенства. Приведем два полезных результата такого типа.

Лемма 2. Пусть $1 \leqslant k \leqslant l$. Тогда

$$
S_{l}(t, p) \geqslant p^{l-k} S_{k}(t, p)
$$

Доказательство. Выберем некоторый многочлен

$$
Q\left(x_{1}, \ldots, x_{k}\right) \in F_{p}\left[x_{1}, \ldots, x_{k}\right]
$$

степени не выше $t$, для которого

$$
\left|W_{\chi}(Q)\right|=S_{k}(t, p)
$$

Рассматривая $Q\left(x_{1}, \ldots, x_{k}\right)$ как многочлен из $F_{p}\left[x_{1}, \ldots, x_{l}\right]$, очевидно, имеем

$$
\left|W_{\chi}(Q)\right|=p^{l-k} S_{k}(t, p) .
$$

Теперь по определению $S_{l}(t, p)$ получаем требуемое неравенство.

Отметим, что лемма 2 фактически используется в некоторых оценках работы [5]. Некоторые оценки величины $S_{l}(t, p)$ можно получить для практически наиболее интересного случая небольших значений $t$. Для этого необходимы только элементарные результаты о числе решений уравнений в конечных полях (см. [2, §1 главы 6]).

Обозначим $S_{l}^{*}(t, p)=\max \left|W_{\chi}(Q)\right|$, где максимум берется по всем многочленам $Q\left(x_{1}, \ldots, x_{l}\right) \in F_{p}\left[x_{1}, \ldots, x_{l}\right]$ степени не выше $t \leqslant l$, принимающим каждое значение из $F_{p}$ хотя бы один раз. Очевидно, что

$$
\begin{aligned}
& S_{l}^{*}(t, p) \leqslant S_{l}(t, p), \\
& S_{l}^{*}(t, 2)=S_{l}(t, 2) .
\end{aligned}
$$


Теорема 7. Справедливы неравенства

$$
\frac{1}{p-1}\left(p^{l}-p^{l-t+1}\right) \leqslant S_{l}^{*}(t, p) \leqslant p^{l}-p^{l-t+1}
$$

Доказательство. Пусть $Q\left(x_{1}, \ldots, x_{l}\right) \in F_{p}\left[x_{1}, \ldots, x_{l}\right]$ - многочлен степени не выше $t \leqslant l$, принимающий каждое значение из $F_{p}$ хотя бы один раз. В соответствии с теоремой $6.11\left[2\right.$, стр. 336] при любом $a \in F_{p}$

$$
N_{a}=\left|\left\{\left(x_{1}, \ldots, x_{l}\right): Q\left(x_{1}, \ldots, x_{l}\right)=a\right\}\right| \geqslant p^{l-t} .
$$

Поэтому

$$
\begin{aligned}
\left|W_{\chi}(Q)\right| & =\left|\sum_{a \in F_{p}} \chi(a) N_{a}\right| \\
& =\left|\sum_{a \in F_{p}} p^{l-t} \chi(a)+\sum_{a \in F_{p}}\left(N_{a}-p^{l-t}\right) \chi(a)\right| \\
& =\left|\sum_{a \in F_{p}}\left(N_{a}-p^{l-t}\right) \chi(a)\right| \\
& \leqslant \sum_{a \in F_{p}}\left(N_{a}-p^{l-t}\right)=p^{l}-p^{l-t+1}
\end{aligned}
$$

откуда следует верхняя оценка для $S_{l}^{*}(t, p)$.

Для доказательства нижней оценки рассмотрим многочлен степени $t$, построенный в примере 6.12 ([2, стр. 337]),

$$
Q\left(x_{1}, \ldots, x_{l}\right)=\operatorname{norm}\left(x_{1}, \ldots, x_{t}\right)
$$

где $\operatorname{norm}\left(x_{1}, \ldots, x_{t}\right)$ - норма элемента поля $F_{p^{t}}$ с координатами $x_{1}, \ldots, x_{t}$ в некотором базисе над $F_{p}$. Хорошо известно (см., например, [1, задача 7 , стр. 34]), что

$$
\left|\left\{\left(x_{1}, \ldots, x_{t}\right): \operatorname{norm}\left(x_{1}, \ldots, x_{t}\right)=a\right\}\right|= \begin{cases}1, & \text { если } a=0 \\ \left(p^{t}-1\right) /(p-1), & \text { если } a \in F_{p}^{*}\end{cases}
$$

Поэтому

$$
N_{a}=\left|\left\{\left(x_{1}, \ldots, x_{l}\right): Q\left(x_{1}, \ldots, x_{l}\right)=a\right\}\right|= \begin{cases}p^{l-t}, & \text { если } a=0 \\ p^{l-t}\left(p^{t}-1\right) /(p-1), & \text { если } a \in F_{p}^{*}\end{cases}
$$

и

$$
\left|W_{\chi}(Q)\right|=\left|\sum_{a \in F_{p}} \chi(a) N_{a}\right|=p^{l-t}\left(\left(p^{t}-1\right) /(p-1)-1\right)=\frac{1}{p-1}\left(p^{l}-p^{l-t+1}\right),
$$

откуда следует нижняя оценка для $S_{l}^{*}(t, p)$. 
При $p=2$

$$
S_{l}^{*}(t, 2)=S_{l}(t, 2)=2^{l}-2^{l-t+1},
$$

что соответствует известному результату из теории двоичных кодов Рида-Маллеpa [9].

Приведем без доказательства верхнюю оценку для $\max \left|W_{\chi}(Q)\right|$ по более широкому классу многочленов, она получается с помощью очевидной модификации доказательства теоремы 6. Введем обозначение

$$
S_{l}^{(d)}(t, p)=\max \left|W_{\chi}(Q)\right|
$$

где максимум берется по всем многочленам $Q\left(x_{1}, \ldots, x_{l}\right) \in F_{p}\left[x_{1}, \ldots, x_{l}\right]$ степени не выше $t \leqslant l$, принимающим каждое из каких-нибудь $p-d$ значений из $F_{p}$ хотя бы один раз. Очевидно, что

$$
S_{l}^{*}(t, p)=S_{l}^{(0)}(t, p) \leqslant S_{l}^{(1)}(t, p) \leqslant \ldots \leqslant S_{l}^{(p-2)}(t, p)=S_{l}(t, p) .
$$

При любом $d=0,1, \ldots$ имеет место оценка

$$
S_{l}^{(d)}(t, p) \leqslant p^{l}-p^{l-t+1}+2 d p^{l-t}
$$

которая нетривиальна (т.е. не превышает $p^{l}$ ) при $d<p / 2$.

\section{4. Суммы Вейля на конечной абелевой группе}

Обозначив $\varphi(x)=\chi(P(x))$, получим функцию $\varphi: F_{q} \rightarrow T$, а сумма Вейля принимает вид

$$
\sum_{x \in F_{q}} \varphi(x) .
$$

При таких обозначениях мы забываем о многочлене $P(x)$ и можем рассматривать всевозможные функции из $F_{q}$ в $T$, а для изучения суммы Вейля привлекать другие (кроме степени многочлена $P(x)$ ) характеристики комплекснозначных функций на конечной абелевой группе. В абстрактном гармоническом анализе сумма

$$
\sum_{x \in G} \varphi(x)
$$

для комплекснозначной функции $\varphi$ на абелевой группе $G$ называется интегралом Хаара (см. [11]). В [12, 13] предложен ряд новых характеристик для таких функций на конечной абелевой группе. Эти характеристики оказались полезными при решении некоторых задач теории кодирования и криптографии. Применим предложенный в работах $[12,13]$ подход к изучению свойств сумм Вейля на конечной абелевой группе.

Через $T^{G}$ обозначим множество функций на $G$ со значениями в $T$. Производная $\frac{d \varphi}{d \sigma}(x)$ функции $\varphi \in T^{G}$ по направлению $\sigma \in G$ определяется равенством

$$
\frac{d \varphi}{d \sigma}(x)=\varphi(x \sigma) \bar{\varphi}(x), \quad x \in G
$$


здесь $\bar{\varphi}(x)$ - число, комплексно сопряженное числу $\varphi(x)$. Множества функций $\mathrm{RM}_{r}(G), \mathrm{RM}_{r}(G) \subseteq \mathrm{RM}_{r+1}(G), r=0,1, \ldots$ на группе $G$ определяются рекуррентным образом:

$$
\begin{aligned}
\operatorname{RM}_{r}(G) & =\left\{\varphi \in T^{G}: \frac{d \varphi}{d \sigma} \in \mathrm{RM}_{r-1}(G), \quad \forall \sigma \in G\right\} \\
\mathrm{RM}_{-1}(G) & =\left\{\varphi \in T^{G}: \varphi(x) \equiv 1\right\} .
\end{aligned}
$$

При этом функции из $\mathrm{RM}_{1}(G)$ - это аффинные функции на $G$, так как они получаются из характеров (линейных функций) умножением на константу: $\varphi \in \mathrm{RM}_{1}(G)$ тогда и только тогда, когда $\varphi(x)=\varphi(e) \chi_{\alpha}(x)$ для некоторого $\alpha \in G$ при всех $x \in G$, здесь $e$ - единичный элемент группы $G$. Функции из $\mathrm{RM}_{2}(G)$ - это квадратичные функции на $G$, так как любая их производная - это аффинная функция.

Связь между интегралами Хаара функции и ее производных задается следующим равенством (см. лемму 3 в [12]):

$$
\left|\sum_{x \in G} \varphi(x)\right|^{2}=\sum_{\sigma \in G} \sum_{x \in G} \frac{d \varphi}{d \sigma}(x) .
$$

Аналогичное соотношение между суммами Вейля многочлена и его производных лежит в основе метода Г. Вейля оценки тригонометрических сумм (см. [14, стр. 89]).

Вычислим значения интегралов Хаара для функций из $\mathrm{RM}_{r}(G)$ при $r=0,1,2$. Очевидно, что если $|G|=q$, то

$$
\sum_{x \in G} \varphi(x)=q c, \quad c \in T \Longleftrightarrow \varphi \in \mathrm{RM}_{0}(G), \quad \varphi(x) \equiv c .
$$

Очевидно, что если $\varphi \in \mathrm{RM}_{1}(G) \backslash \mathrm{RM}_{0}(G)$, то

$$
\sum_{x \in G} \varphi(x)=0
$$

Пусть теперь $\varphi \in \mathrm{RM}_{2}(G)$. Тогда

$$
\frac{d \varphi}{d \sigma} \in \mathrm{RM}_{1}(G)
$$

для всех $\sigma \in G$. Из результатов работы [13] вытекает, что при этом множество

$$
\mathscr{J}(\varphi)=\left\{\sigma \in G: \frac{d \varphi}{d \sigma} \in \mathrm{RM}_{0}(G)\right\}
$$

является подгруппой группы $G$, a

$$
\frac{d \varphi}{d \sigma}(x) \equiv c_{\varphi}(\sigma)
$$

при $\sigma \in \mathscr{J}(\varphi)$ является характером подгруппы $\mathscr{J}(\varphi)$. Используя теперь равенство (11), получаем следующее утверждение. 
Предложение 3. Пусть $\varphi \in \mathrm{RM}_{2}(G)$. Тогда

$$
\left|\sum_{x \in G} \varphi(x)\right|= \begin{cases}\sqrt{|\mathscr{J}(\varphi)|} q^{1 / 2}, & \text { если характер } c_{\varphi} \text { тривиален на подгруппе } \mathscr{J}(\varphi) \\ 0 & \text { в противном случае. }\end{cases}
$$

Чтобы применять полученные результаты в случае конечного поля, надо научиться описывать многочлены $P(x)$ такие, что

$$
\chi(P(x)) \in \mathrm{RM}_{r}\left(F_{q}\right), \quad r=0,1,2 .
$$

Пусть $G$ - конечная мультипликативная абелева группа порядка $q=p_{1}^{k_{1}} \ldots p_{s}^{k_{s}}$, $p_{1}, \ldots, p_{s}-$ простые числа и $G=G_{1} \times \ldots \times G_{s}$ - ее представление в виде прямого произведения циклических групп $G_{1}, \ldots, G_{s}$ порядков $q_{1}=p_{1}^{k_{1}}, \ldots, q_{s}=p_{s}^{k_{s}}$ соответственно. Пусть $\eta_{1}, \ldots, \eta_{s}$ - порождающие элементы групп $G_{1}, \ldots, G_{s}$, а наибольшее общее кратное $m=\operatorname{LCM}\left(q_{1}, \ldots, q_{s}\right)$ - экспонента группы $G$. Хорошо известно [1, cтр. 39], что каждый характер $\chi$ группы $G$ имеет вид

$$
\chi_{\alpha_{1}, \ldots, \alpha_{s}}\left(\eta_{1}^{x_{1}} \ldots \eta_{s}^{x_{s}}\right)=\exp \left(2 \pi i\left(\alpha_{1} x_{1}\right) / q_{1}\right) \ldots \exp \left(2 \pi i\left(\alpha_{s} x_{s}\right) / q_{s}\right)
$$

при некоторых $\alpha_{j}=0,1, \ldots, q_{j}-1,1 \leqslant j \leqslant s$. Для дальнейшего удобно представить характер в виде

$$
\chi_{\alpha_{1}, \ldots, \alpha_{s}}\left(\eta_{1}^{x_{1}} \ldots \eta_{s}^{x_{s}}\right)=\exp \left((2 \pi i / m)\left(\alpha_{1}^{\prime} x_{1}+\ldots+\alpha_{s}^{\prime} x_{s}\right)\right),
$$

где $\alpha_{j}^{\prime}=\left(m / q_{j}\right) \alpha_{j}, j=1, \ldots, s$.

Поскольку каждый элемент группы $G$ однозначно представим в виде $\eta_{1}^{x_{1}} \ldots \eta_{s}^{x_{s}}$ и при умножении элементов группы показатели $x_{1}, \ldots, x_{s}$ складываются, будем отождествлять группу $G$ с прямым произведением $Z_{q_{1}} \times \ldots \times Z_{q_{s}}$, где $Z_{q_{j}}$ - кольцо вычетов по модулю $q_{j}$.

Определим множества

$$
M_{r}(G) \subset T^{G}, \quad M_{r}(G) \subseteq M_{r+1}(G), \quad r=0,1, \ldots,
$$

функций на группе $G$ следующим образом:

$$
\begin{aligned}
M_{r}(G) & = \\
& =\left\{\exp \left((2 \pi i / m) P\left(x_{1}, \ldots, x_{s}\right)\right): P\left(x_{1}, \ldots, x_{s}\right) \in Z_{m}\left[x_{1}, \ldots, x_{s}\right], \operatorname{deg} P \leqslant r\right\} .
\end{aligned}
$$

Лемма 3. Множества функций $M_{r}(G), r=0,1, \ldots$, удовлетворяют рекуррентному соотношению

$$
M_{r}(G)=\left\{\varphi \in T^{G}: \frac{d \varphi}{d \sigma} \in M_{r-1}(G), \forall \sigma \in G\right\} .
$$

Доказательство. Пусть $\varphi\left(x_{1}, \ldots, x_{s}\right) \in M_{r}(G)$. По определению производной при любом $\sigma=\left(\sigma_{1}, \ldots, \sigma_{s}\right) \in G$

$$
\begin{aligned}
\frac{d \varphi}{d \sigma}(x) & =\varphi(x+\sigma) \bar{\varphi}(x) \\
& \left.=\exp ((2 \pi i) / m)\left(P\left(x_{1}+\sigma_{1}, \ldots, x_{s}+\sigma_{s}\right)-P\left(x_{1}, \ldots, x_{s}\right)\right)\right),
\end{aligned}
$$


для всех $x \in G$.

Пользуясь свойствами многочленов, легко доказать, что

$$
\operatorname{deg}\left(P\left(x_{1}+\sigma_{1}, \ldots, x_{s}+\sigma_{s}\right)-P\left(x_{1}, \ldots, x_{s}\right)\right) \leqslant \operatorname{deg} P\left(x_{1}, \ldots, x_{s}\right)-1
$$

для любого $\sigma \in G$; найдется такое $\sigma^{0} \in G$, что

$$
\operatorname{deg}\left(P\left(x_{1}+\sigma_{1}^{0}, \ldots, x_{s}+\sigma_{s}^{0}\right)-P\left(x_{1}, \ldots, x_{s}\right)\right)=\operatorname{deg} P\left(x_{1}, \ldots, x_{s}\right)-1 .
$$

Дальнейшее доказательство леммы 3 очевидно.

Теорема 8. Для любой конечной абелевой группы $G$

$$
\mathrm{RM}_{r}(G) \subseteq M_{r}(G), \quad r=1,2, \ldots
$$

Доказательство. Известно, что функции из $\mathrm{RM}_{1}(G)$ - это характеры, умноженные на константу. Учитывая общий вид характеров $(12)$ и определение $M_{1}(G)$, заключаем, что

$$
\mathrm{RM}_{1}(G) \subseteq M_{1}(G)
$$

В силу леммы 3 множества $M_{r}(G)$ удовлетворяют тому же рекуррентному соотношению, что и $\mathrm{RM}_{r}(G)$. Поэтому из (13) по индукции получаем утверждение теоремы 8.

Заметим, что в общем случае включение (13) нельзя усилить и заменить на равенство

$$
\mathrm{RM}_{1}(G)=M_{1}(G)
$$

поскольку в выражении (12) для характеров есть еще дополнительное условие

$$
\alpha_{j}^{\prime}=\frac{m}{q_{j}} \alpha_{j}, \quad j=1, \ldots, s .
$$

Это условие означает, что коэффициенты $a_{j} \in Z_{m}, j=1, \ldots, s$, линейной функции из $Z_{m}\left[x_{1}, \ldots, x_{s}\right]$

$$
a_{1} x_{1}+\ldots+a_{s} x_{s}
$$

должны делиться на целье числа $m / q_{j}, j=1, \ldots, s$ соответственно.

Из этого замечания и доказательства теоремы 8 легко получить следующее утверждение.

Теорема 9. Пусть для конечной абелевой группы $G$ выполнено равенство (14). Тогда

$$
\mathrm{RM}_{r}(G)=M_{r}(G)
$$

$\partial л я$ всех $r=1,2, \ldots$

Приведем два примера групп, для которых указанное выше условие на коэффициенты

$$
\alpha_{j}^{\prime}=\frac{m}{q_{j}} \alpha_{j}, \quad j=1, \ldots, s,
$$

выполняется автоматически, а поэтому выполнено равенство (14) и теорема 9. Это группы, для которых $q_{1}=q_{2}=\ldots=q_{s}=m=p$, где $p$ - некоторое простое число, т. е. элементарные абелевы $p$-группы, и группы, для которых $s=1$ и поэтому $m=q_{1}$, т. е. циклические группы.

Отсюда очевидным образом получается описание $\mathrm{RM}_{r}(G)$ для аддитивной и мультипликативной групп конечного поля. 
Следствие 2. Пустъ $G$ - аддитивная группа конечного поля $F_{q}, q=p^{l}$, каждый элемент которой представлен в виде

$$
\left(x_{1}, \ldots, x_{l}\right), \quad x_{j} \in F_{p}, \quad j=1, \ldots, l .
$$

Тогда для $r=1,2, \ldots$

$$
\mathrm{RM}_{r}(G)=\left\{\exp \left((2 \pi i / p) P\left(x_{1}, \ldots, x_{l}\right)\right): P\left(x_{1}, \ldots, x_{l}\right) \in F_{p}\left[x_{1}, \ldots, x_{l}\right], \operatorname{deg} P \leqslant r\right\} .
$$

Следствие 3. Пусть $G$ - мулътипликативная группа конечного поля $F_{q}, q=p^{l}$, каждый элемент которой представлен в виде $\eta^{x}, x \in Z_{q-1}, \eta$ - примитивный элемент поля. Тогда для $r=1,2, \ldots$

$$
\mathrm{RM}_{r}(G)=\left\{\exp ((2 \pi i /(q-1)) P(x)): P(x) \in Z_{q-1}[x], \operatorname{deg} P \leqslant r\right\} .
$$

В [13] описаны кусочно-линейные и кусочно-аффинные функции на группе. Для них интегралы Хаара также вычисляются легко.

Пусть $G=U \times V, \rho: V \rightarrow U, \xi \in T^{V}$. Функция $\varphi \in T^{G}$, представимая в виде

$$
\varphi(y, z)=\chi_{\rho(z)}(y), \quad y \in U, \quad z \in V,
$$

называется кусочно-линейной, а представимая в виде

$$
\varphi(y, z)=\xi(z) \chi_{\rho(z)}(y), \quad y \in U, \quad z \in V,
$$

называется кусочно-аффинной. Для данной функции $\varphi \in T^{G}$ может быть несколько различных представлений в кусочно-линейной или кусочно-аффинной форме. При любом фиксированном $z=z^{0} \in V$ функция $\varphi\left(y, z^{0}\right)$ аффинна. Из результатов работы [13] (см. лемму 4) вытекают следующие формулы для интегралов Хаара:

$$
\begin{gathered}
\sum_{(y, z) \in G} \chi_{\rho(z)}(y)=|U|\left|\rho^{-1}(e)\right|, \\
\sum_{(y, z) \in G} \xi(z) \chi_{\rho(z)}(y)=|U| \sum_{z: \rho(z)=e} \xi(z) .
\end{gathered}
$$

Чтобы применять полученные результаты в случае конечного поля, надо научиться представлять функции $\chi(P(x))$ (для различных многочленов $P(x)$ ) в кусочноаффинной или кусочно-линейной форме.

Интересно, что кусочно-линейные функции на аддитивной группе конечного поля возникают в процессе доказательства оценки Вейля, а точнее, в процессе вычисления коэффициентов $L$-функции Артина. Полезно разобрать этот пример более подробно.

Пример 2. Многочлен $\widetilde{g}\left(u_{1}, \ldots, u_{\nu}\right) \in F_{q}\left[u_{1}, \ldots, u_{\nu}\right]$ задается формулой (в обозначениях книги [1], см. формулу (7), стр. 44)

$$
\tilde{g}\left(u_{1}, \ldots, u_{\nu}\right)=\sum_{\mu=1}^{n} b_{n-\mu} \sum_{\lambda_{1}+2 \lambda_{2}+\ldots+\nu \lambda_{\nu}=\mu} a_{\lambda_{1}, \ldots, \lambda_{\nu}}^{(\mu)} u_{1}^{\lambda_{1}} \ldots u_{\nu}^{\lambda_{\nu}}
$$

где $b_{0}, \ldots, b_{n-1} \in F_{q}, a_{\lambda_{1}, \ldots, \lambda_{\nu}}^{(\mu)}$ - целые числа, причем

$$
a_{0, \ldots, 0,1,0, \ldots, 0,1,0, \ldots, 0}^{(n)}=(-1)^{n-2} n .
$$

Подробно о многочлене $\widetilde{g}$ и его применении при доказательстве оценки Вейля можно прочитать в [1, стр. 42-47]. 
Пусть $\nu \leqslant n, m=\lceil n / 2\rceil+1$. При $\nu \geqslant m$ положим $z_{j}=u_{j}, j=1, \ldots, m-1$, $y_{k}=u_{m-1+k}, k=1, \ldots, \nu-m+1$ и рассмотрим функцию

$$
\varphi(y, z)=\chi\left(\widetilde{g}\left(u_{1}, \ldots, u_{\nu}\right)\right)=\chi\left(\widetilde{g}\left(z_{1}, \ldots, z_{m-1}, y_{1}, \ldots, y_{\nu-m+1}\right)\right),
$$

где $\chi$ - нетривиальный аддитивный характер поля $F_{q}$. Покажем, что при любой фиксации $z=z^{0}$ функция $\varphi\left(y, z^{0}\right)$ аффинная по $y$, т. е. функция $\varphi(y, z)-$ кусочноаффинная. В соответствии с определением в многочлен $\widetilde{g}\left(u_{1}, \ldots, u_{\nu}\right)$ могут входить только одночлены $u_{1}^{\lambda_{1}} \ldots u_{\nu}^{\lambda_{\nu}}$ специального вида, который задается уравнением в целых числах на допустимые значения $\lambda_{1}, \ldots, \lambda_{\nu}$ :

$$
\lambda_{1}+2 \lambda_{2}+\ldots+\nu \lambda_{\nu}=\mu, \quad \mu=1, \ldots, n .
$$

Следующие свойства решений этого уравнения проверяются непосредственно.

Для всех $j \geqslant m$ справедливы неравенства $\lambda_{j} \leqslant 1$.

Если $\lambda_{j}=1$ при некотором $j \geqslant m$, то

$$
\lambda_{j+1}=\ldots=\lambda_{\nu}=0, \quad \lambda_{n-j+1}=\ldots=\lambda_{j-1}=0 .
$$

Если $\lambda_{j}=\lambda_{n-j}=1$ при некотором $j \geqslant m$, то решение единственно: $\mu=n$, остальные $\lambda_{k}$ равны нулю. Коэффициент при этом одночлене $u_{j} u_{n-j}$ равен $(-1)^{n-2} n b_{0}$.

Зафиксируем теперь некоторое $j \geqslant m$ и соберем в многочлене $\widetilde{g}\left(u_{1}, \ldots, u_{\nu}\right)$ все одночлены, в которые входит $u_{j}$. Учитывая приведенные выше свойства, мы получим сумму

$$
u_{j}\left(c u_{n-j}+P_{n-j-1}\left(u_{1}, \ldots, u_{n-j-1}\right)\right),
$$

где $c=(-1)^{n-2} n b_{0}$, a $P_{n-j-1}\left(u_{1}, \ldots, u_{n-j-1}\right)$ - некоторый многочлен. Таким образом, многочлен $\widetilde{g}\left(u_{1}, \ldots, u_{\nu}\right)$ представляется в виде

$$
\begin{aligned}
\widetilde{g}\left(u_{1}, \ldots, u_{\nu}\right)=u_{\nu} & \left(c u_{n-\nu}+P_{n-\nu-1}\left(u_{1}, \ldots, u_{n-\nu-1}\right)\right) \\
& +u_{\nu-1}\left(c u_{n-\nu+1}+P_{n-\nu}\left(u_{1}, \ldots, u_{n-\nu}\right)\right) \ldots \\
& +u_{m}\left(c u_{n-m}+P_{n-m-1}\left(u_{1}, \ldots, u_{n-m-1}\right)\right)+P_{n-m}\left(u_{1}, \ldots, u_{m-1}\right) .
\end{aligned}
$$

Отсюда видно, что $\widetilde{g}\left(u_{1}, \ldots, u_{\nu}\right)$ при любой фиксации переменных $u_{1}, \ldots, u_{m-1}$ является аффинной функцией от переменных $u_{m}, \ldots, u_{\nu}$. Значит, и функция $\varphi(y, z)$ кусочно-аффинная, причем разветвляющее отображение $\rho: F_{q^{n-m}} \rightarrow F_{q^{\nu-m+1}}$ задается следующими координатными функциями:

$$
\begin{gathered}
c u_{n-\nu}+P_{n-\nu-1}\left(u_{1}, \ldots, u_{n-\nu-1}\right), \\
c u_{n-\nu+1}+P_{n-\nu}\left(u_{1}, \ldots, u_{n-\nu}\right), \\
\ldots \\
c u_{n-m}+P_{n-m-1}\left(u_{1}, \ldots, u_{n-m-1}\right) .
\end{gathered}
$$

Отметим, что в случае нечетного $n$ и $\nu=n-1$ можно продолжить аналогичные рассуждения дальше и получить кусочно-аффинное представление многочлена $\tilde{g}\left(u_{1}, \ldots, u_{n-1}\right)$ от половины своих переменных.

Авторы благодарны Л. А. Бассалыго и В. А. Зиновьеву за интересные обсуждения и полезные замечания, способствовавшие улучшению работы. 


\section{Список литературы}

1. Степанов С. А. Арифметиха алгебрачческих кривых. Наука, Москва, 1991.

2. Лидл Р., Нидеррайтер Г. Конечные поля, т. 1, 2, Мир, Москва, 1988.

3. Schmidt W. M. Equations over finite fields. An elementary approach. Lect. Notes Math. (1976) 536.

4. Виноградов И. М. Метод тригометрических сумм в теории чисел. Наука, Москва, 1971.

5. Бассалыго Л. А., Зиновьев В. А., Лицын С. Н. Оценка снизу полных тригонометрических сумм через кратные суммы. Докљ. АН СССР (1988) 300, №5, 1033-1036.

6. Степанов С. А. О нижних оценках сумм характеров над конечными полями. Дискретная математика (1991) 3, №2, 77-86.

7. Глухов М. М. Нижние оценки сумм характеров от многочленов над конечными полями. Дискретнал математика (1994) 6, №3, 136-142.

8. Бассалыго Л. А., Зиновьев В. А. Многочлены специального вида над конечным полем с максимальным модулем тригонометрической суммы. Успехи матем. наук (1997) 52 , №2 (314), 31-44.

9. Мак-Вильямс Ф. Дж., Слоэн Н. Дж. Теория кодов, исправляющих ошибки. Связь, Москва, 1979.

10. Moreno O., Zinoviev V. A., Kumar P. V. An extension of the Weil-Carlitz-Uchiyama bound. Finite Fields and their Appl. (1995) 1, №3, 360-371.

11. Хьюитт Э., Росс К. Абстрактный гармонический анализ. Структура топологических групп. Теория интегрирования. Представления групп, т. 1, Наука, Москва, 1975.

12. Логачев О. А., Ященко В. В. Коды типа Рида-Маллера на конечной абелевой группе. Пробл. передачи информачии (1998) 34, №2, 32-46.

13. Логачев О. А., Сальников А. А., Ященко В. В. Бент-функции на конечной абелевой группе. Дискретная математика (1997) 9, №4, 3-20.

14. Коробов Н. М. Тригонометрические суммы и их приложения. Наука, Москва, 1989.

Статья поступила 15.02.1999. 